\title{
Logistic Regression Type Small Area Estimations Based on Relative Error
}

\author{
Hee-Jin Hwang ${ }^{1} \cdot$ Key-II Shin ${ }^{2}$ \\ ${ }^{1}$ Data Information Center, NHN; ${ }^{2}$ Department of Statistics, Hankuk University of Foreign Studies
}

(Received January 2011; accepted March 2011)

\begin{abstract}
Almost all small area estimations are obtained by minimizing the mean squared error. Recently relative error prediction methods have been developed and adapted to small area estimation. Usually the estimators obtained by using relative error prediction is called a shrinkage estimator. Especially when data set consists of large range values, the shrinkage estimator is known as having good statistical properties and an easy interpretation. In this paper we study the shrinkage estimators based on logistic regression type estimators for small area estimation. Some simulation studies are performed and the Economically Active Population Survey data of 2005 is used for comparison.
\end{abstract}

Keywords: Shrinkage estimator, mean squared error, logistic mixed model, logistic regression model.

\section{Introduction}

Most sample surveys are designed to produce estimates for a whole geographical area. The sample sizes of small areas such as cities and counties would be small (even zeros for some areas) because the overall sample size in a survey is usually determined to provide a specific accuracy at a higher level of aggregation than that of a small area. In that case, sample proportions such as unemployment rates in counties may be poorly estimated. Usually survey estimates based on such small sample sizes could provide formidable standard errors leading to unacceptable confidence intervals. However, a heavy burden of time and cost occurs in obtaining an acceptable accuracy for the statistic that of a small area. Therefore, instead of doing the extra survey overcoming the unacceptable accuracy caused by small sample size, reliable official statistics for the small areas can be produced efficiently by applying the small area estimation methods.

The small area estimation methods can be classified as design-based and model-based. In general, when the auxiliary information is available, model-based methods are known better. Especially, for binary data such as unemployment data, the logistic regression estimator and logistic mixed estimator are known as having good statistical properties. In addition, the random effects models that treat each county as a cluster can provide improved estimates. Studies on the logistic regression

This research was supported by Basic Science Research Program through the National Research Foundation of Korea(NRF) funded by the Ministry of Education, Science and Technology(2009-0072926).

${ }^{2}$ Corresponding author: Professor, Department of Statistics, Hankuk University of Foreign Studies, Yongin, Kyonggi 449-791, Korea. E-mail: keyshin@hufs.ac.kr 
type estimators to estimate unemployment rates can be found in Kim and Choi (2004) and Yeo et al. (2008).

In this study, we suggest estimators obtained by minimizing relative error(RE) or mean squared percentage error(MSPE) instead of minimizing MSE. This estimator has the advantage of an easy calculation obtained by multiplying a certain constant term to the original estimator obtained by minimizing MSE. The estimator known as a shrinkage estimator was studied by Hwang and Shin (2008, 2009); however, in those papers, the application of logistic regression estimator and logistic mixed estimator to small area estimation was not performed in their papers. Hence the applicability of a shrinkage estimation method to logistic regression type estimators should be studied and the superiority of shrinkage small area estimators in an aspect of relative error criterion should be examined.

In this paper we compare the efficiency of each estimator such as direct estimator, logistic regression estimator, logistic mixed estimator, and the shrinkage type estimators derived from logistic type estimators. In Section 2, we briefly explain logistic regression type small area estimators which are practically and widely used for binary data. In addition, the shrinkage estimators made from the original small area estimators are explained. Section 3 performs the analysis of data using the estimators mentioned before and the results for comparison of the estimators are shown. For comparison, we use the unemployment data from the Monthly Report on the Economically Active Population Survey in Korea (2005). Section 4 includes some concluding remarks.

\section{Suggested Methods for Small Area Estimations}

In this section, we briefly summarize some small area estimators mentioned in Section 1. Some widely used estimators for binary data obtained by minimizing MSE are explained and we study the new shrinkage estimators obtained by using RE.

\subsection{Small area estimators using MSE}

Some small area estimation methods have been suggested as design-based estimation such as direct estimation, synthetic estimation, and composite estimation. In addition, the well-known model based estimations have been suggested such as regression estimation. Empirical Bayes estimation(EB), Hierarchical Bayes estimation(HB). For binary response data, logistic regression type estimators are widely used. Therefore, in this study, we consider two logistic regression type estimators specially used for binary data: the logistic regression estimator and the logistic mixed estimator. Details on these estimators can be found in Agresti (2002) and Rao (2003).

2.1.1. Direct estimator The direct estimator, $\hat{Y}_{D E}$ is defined as $\hat{Y}_{D E}=\hat{Y}_{i}=\sum_{j} w_{i j} y_{i j}$ where $\hat{Y}_{i}$ is the estimate of the interesting variable in $i^{\text {th }}$ small area, $w_{i j}, i=1, \ldots, n ; j=1, \ldots, n_{i}$ is the sampling weight and $y_{i j}$ is the value of $j^{\text {th }}$ element in $i^{\text {th }}$ small area. Usually the sampling weight $w_{i j}$ have the same value in the same stratum. Hence for simplicity, we use $w_{i j}=1$ for all $i$ and $j$ throughout this paper. This constant sampling weight does not affect the comparison result of the estimators' superiority.

2.1.2. Logistic regression estimator The logistic regression model is defined by following.

$$
\log \left(\frac{p_{i j}(x)}{1-p_{i j}(x)}\right)=\beta_{0}+\beta_{1} x_{1 i j}+\beta_{2} x_{2 i j}+\cdots+\beta_{p} x_{p i j}
$$


Here $p_{i j}=P\left(y_{i j}=1 \mid x_{1 i j}, x_{2 i j}, \ldots, x_{p i j}\right), i=1, \ldots, n ; j=1, \ldots, n_{i}$ and $x_{k i j}$ 's are independent variables. In addition, we use the common parameters $\beta_{i}^{\prime} s$ through the whole areas.

The estimates of the sample proportion, $\hat{p}_{i j}$ are defined as

$$
\log \left(\frac{\hat{p}_{i j}(x)}{1-\hat{p}_{i j}(x)}\right)=\hat{\beta}_{0}+\hat{\beta}_{1} x_{1 i j}+\hat{\beta}_{2} x_{2 i j}+\cdots+\hat{\beta}_{p} x_{p i j}
$$

where $\hat{p}_{i j}=\hat{P}\left(y_{i j}=1 \mid x_{1 i j}, x_{2 i j}, \ldots, x_{p i j}\right)$ and the estimates of $\beta_{i}^{\prime} s$ are easily obtained by using SAS/GENMOD procedure.

Then the logistic regression small estimator, $\hat{Y}_{L O G I T}$, is defined as

$$
\hat{Y}_{L O G I T}=\hat{Y}_{i}=\sum_{j=1}^{n_{i}} \hat{y}_{i j}=\sum_{j=1}^{n_{i}} \hat{p}_{i j} .
$$

2.1.3. Logistic mixed estimator The logistic mixed model is defined by following.

$$
\log \left(\frac{p_{i j}(x)}{1-p_{i j}(x)}\right)=\beta_{0}+\beta_{1} x_{1 i j}+\cdots+\beta_{p} x_{p i j}+v_{i} .
$$

Here $p_{i j}=P\left(y_{i j}=1 \mid x_{1 i j}, x_{2 i j}, \ldots, x_{p i j}\right), i=1, \ldots, n ; j=1, \ldots, n_{i}$ and $v_{i}$ is the random effect about the differences of small areas. In addition, $x_{k i j}$ 's and $\beta_{i}$ 's basic assumptions are the same as those in the logistic regression model.

Then the estimates of the sample proportion, $\hat{p}_{i j}$ are obtained by

$$
\log \left(\frac{\hat{p}_{i j}(x)}{1-\hat{p}_{i j}(x)}\right)=\hat{\beta}_{0}+\hat{\beta}_{1} x_{1 i j}+\cdots+\hat{\beta}_{p} x_{p i j}+\hat{v}_{i}
$$

and the estimates of $\beta_{i}^{\prime} s$ and $v_{i}^{\prime} s$ can be obtained by using SAS/GLIMMIX procedure. Finally the logistic mixed estimator, $\hat{Y}_{L M M}$, is defined as

$$
\hat{Y}_{L M M}=\hat{Y}_{i}=\sum_{j=1}^{n_{i}} \hat{y}_{i j}=\sum_{j=1}^{n_{i}} \hat{p}_{i j} .
$$

\subsection{Small area estimator using $R E$}

2.2.1. Shrinkage estimator: $\hat{\boldsymbol{Y}}^{S \boldsymbol{H}}$ Most of the proposed small area estimation methods have been obtained by minimizing MSE, which is $E(Y-\hat{Y})^{2}$. It is very ordinary to use this criterion under the model assumption of homogeneous error variance; however, the homogeneous error variance sometimes does not provide a reasonable interpretation in terms of the relative error criterion for a small value of data. In this case, it may be desirable to use the criterion of RE or MSPE instead of MSE. See more details on RE prediction in Park and Stefanski (1997) and Hwang and Shin (2008, 2009).

The predictor using RE criterion is obtained by

$$
\min E\left(\frac{Y-g^{*}}{Y}\right)^{2}
$$

and the best relative error predictor $g^{*}$ is defined as:

$$
g^{*}=\frac{E(1 / Y)}{E\left(1 / Y^{2}\right)} .
$$


We suppose the following assumptions on mean and variance as in Jeong and Shin (2008).

$$
\left(\frac{Y-\mu}{\mu}\right)^{m}=o_{p}(1), \quad m=2,3, \ldots, \mu=E(Y) .
$$

Then taking the expectations of the Taylor expansion of $Y^{-k}, k=1,2$, we have the following results.

$$
\begin{gathered}
E\left(\frac{1}{Y}\right) \approx \mu^{-1}\left(1+\mathrm{CV}^{2}\right) \\
E\left(\frac{1}{Y^{2}}\right) \approx \mu^{-2}\left(1+3 \mathrm{CV}^{2}\right)
\end{gathered}
$$

Here CV is a coefficient of variation. By plugging (2.8) into (2.7) we have

$$
\frac{E(1 / Y)}{E\left(1 / Y^{2}\right)} \approx \frac{\mu^{-1}\left(1+\mathrm{CV}^{2}\right)}{\mu^{-2}\left(1+3 \mathrm{CV}^{2}\right)}=\mu \frac{\left(1+\mathrm{CV}^{2}\right)}{\left(1+3 \mathrm{CV}^{2}\right)}
$$

Replacing $\mu$ with $\hat{Y}$, we have the following shrinkage estimator.

$$
\hat{Y}^{S H}=\hat{Y} \frac{\left(1+\widehat{\mathrm{CV}}^{2}\right)}{\left(1+3 \widehat{\mathrm{CV}}^{2}\right)} .
$$

See more details on derivations in Jeong and Shin (2008) and Hwang and Shin (2009). This formula shows that $\hat{Y}^{S H}<\hat{Y}$ for $\widehat{\mathrm{CV}}^{2}>0$. Hence we have the 'shrinkage estimator' and we will use this as a small area estimator. Here $\widehat{\mathrm{CV}}$, the estimate of $\mathrm{CV}=\sigma / \mu$ can be estimated by $s / \bar{Y}$.

2.2.2. Suggested shrinkage estimator In this study, we suggest two shrinkage estimators, logistic regression shrinkage estimator $\hat{Y}_{L O G I T}^{S H}$, and logistic mixed shrinkage estimator $\hat{Y}_{L M M}^{S H}$, based on (2.3) and (2.6) with (2.9).

$$
\hat{Y}_{L O G I T}^{S H}=\hat{Y}_{L O G I T} \frac{\left(1+\widehat{\mathrm{CV}}^{2}\right)}{\left(1+3 \widehat{\mathrm{CV}}^{2}\right)}, \quad \hat{Y}_{L M M}^{S H}=\hat{Y}_{L M M} \frac{\left(1+\widehat{\mathrm{CV}}^{2}\right)}{\left(1+3 \widehat{\mathrm{CV}}^{2}\right)} .
$$

The estimators $\hat{Y}_{L O G I T}$ and $\hat{Y}_{L M M}$ are easily obtained using SAS. Also estimates of mean and variance, $\bar{Y}$ and $S^{2}$ are calculated in each small area to obtain $\widehat{\mathrm{CV}}$. So $\hat{Y}_{L O G I T}^{S H}, \hat{Y}_{L M M}^{S H}$ are easily calculated.

\section{Data Analysis and Simulation}

For data analysis, we consider three estimators, $\hat{Y}_{D E}, \hat{Y}_{L O G I T}$ and $\hat{Y}_{L M M}$ introduced in Section 2.1 ; in addition, as the shrinkage estimators, we consider $\hat{Y}_{L O G I T}^{S H}$ and $\hat{Y}_{L M M}^{S H}$ defined in Section 2.2. However, for comparison, we use four model-based estimators, $\hat{Y}_{L O G I T}, \hat{Y}_{L M M}, \hat{Y}_{L O G I T}^{S H}$ and $\hat{Y}_{L M M}^{S H}$.

\subsection{Data analysis}

In this paper, we use the unemployment data from the Monthly Report on the Economically Active Population Survey in Korea (2005) to compare the efficiency of shrinkage estimators and the others. 
Table 3.1. Estimates of the total number of unemployed persons

\begin{tabular}{|c|c|c|c|c|c|}
\hline area code & $\hat{Y}_{D E}$ & $\hat{Y}_{L O G I T}$ & $\hat{Y}_{L M M}$ & $\hat{Y}_{L O G I T}^{S H}$ & $\hat{Y}_{L M M}^{S H}$ \\
\hline 31011 & 11674 & 4826 & 5610 & 4623 & 4535 \\
\hline 31012 & 4746 & 5983 & 5744 & 5786 & 5118 \\
\hline 31013 & 4542 & 4387 & 4455 & 4232 & 3870 \\
\hline 31021 & 7466 & 6394 & 6423 & 6175 & 5585 \\
\hline 31022 & 4967 & 6266 & 5993 & 6062 & 5307 \\
\hline 31023 & 3330 & 5218 & 5028 & 4983 & 4369 \\
\hline 31030 & 10888 & 8706 & 9071 & 8449 & 7950 \\
\hline 31041 & 6463 & 5266 & 5347 & 5110 & 4699 \\
\hline 31042 & 8695 & 5363 & 5631 & 5180 & 4770 \\
\hline 31051 & 3281 & 9244 & 8354 & 8945 & 7272 \\
\hline 31052 & 2129 & 4279 & 3995 & 4091 & 3555 \\
\hline 31053 & 3523 & 3859 & 3742 & 3703 & 3199 \\
\hline 31060 & 12383 & 6786 & 7637 & 6581 & 6611 \\
\hline 31070 & 11346 & 7492 & 8493 & 7310 & 7683 \\
\hline 31080 & 4097 & 1833 & 1981 & 1728 & 1567 \\
\hline 31090 & 16319 & 12154 & 12392 & 11807 & 10969 \\
\hline 31101 & 7272 & 7392 & 7396 & 7157 & 6397 \\
\hline 31102 & 12135 & 9249 & 9757 & 8998 & 8505 \\
\hline 31110 & 412 & 671 & 648 & 637 & 563 \\
\hline 31120 & 2776 & 4316 & 4112 & 4141 & 3617 \\
\hline 31130 & 6175 & 11637 & 10230 & 11391 & 9077 \\
\hline 31140 & 1954 & 2538 & 2478 & 2448 & 2161 \\
\hline 31150 & 5417 & 7489 & 7524 & 7216 & 6477 \\
\hline 31170 & 1502 & 3105 & 2958 & 2974 & 2618 \\
\hline 31180 & 4431 & 3252 & 3336 & 3084 & 2756 \\
\hline 31190 & 14817 & 13664 & 13928 & 13387 & 12713 \\
\hline 31200 & 4638 & 4672 & 4668 & 4476 & 3903 \\
\hline 31210 & 1289 & 3199 & 3010 & 3080 & 2661 \\
\hline 31220 & 2859 & 2690 & 2670 & 2582 & 2254 \\
\hline 31230 & 1979 & 3618 & 3444 & 3491 & 3070 \\
\hline 31240 & 2795 & 6355 & 5847 & 6141 & 5114 \\
\hline 31260 & 2893 & 3990 & 3863 & 3836 & 3381 \\
\hline 31270 & 3326 & 2965 & 2960 & 2843 & 2558 \\
\hline 31380 & 1318 & 1390 & 1332 & 1326 & 1135 \\
\hline
\end{tabular}

Some small areas having all "0" values of the interesting variable are excluded in this analysis. Therefore, the final data used in this analysis consists of 34 small areas and 4916 observations. We calculate the sample means and variances in each small area with given samples and obtain the shrinkage estimators. The data set consists of binary response values such as "employed or not", and the explanatory variable such as administrative district code, gender, level of education, age, and type of housing. Since two variables, level of education $\left(X_{1}\right)$ and age $\left(X_{2}\right)$ are statistically significant, we include these two variables as explanatory variables. For 34 small areas, we calculate five small area estimators, $\hat{Y}_{D E}, \hat{Y}_{L O G I T}, \hat{Y}_{L M M}, \hat{Y}_{L O G I T}^{S H}$ and $\hat{Y}_{L M M}^{S H}$ with whole data. The results of estimates are shown in Table 3.1.

As mentioned before, the data-based estimator, $\hat{Y}_{D E}$ is an unbiased estimator; however, it has a large variance. In several small areas, the estimated values of $\hat{Y}_{D E}$ are quite different from the other model based estimates. Especially in small areas coded by 31022, 31060, 31070, $\hat{Y}_{D E}$ has larger values than the others. On the contrary, in small areas coded by $31023,31052,31120,31210$, the 
Table 3.2. Correlation results with $\hat{Y}_{D E}$

\begin{tabular}{ccccc}
\hline & $\hat{Y}_{L O G I T}$ & $\hat{Y}_{L M M}$ & $\hat{Y}_{L O G I T}^{S H}$ & $\hat{Y}_{L M M}^{S H}$ \\
\hline correlation coefficient & 0.75259 & 0.83137 & 0.75270 & 0.82156 \\
\hline
\end{tabular}

results are reversed. The comparison of $\hat{Y}_{L O G I T}$ with $\hat{Y}_{L O G I T}^{S H}$ shows that all estimates of $\hat{Y}_{L O G I T}$ are larger than $\hat{Y}_{L O G I T}^{S H}$ as expected. The comparison between $\hat{Y}_{L M M}$ and $\hat{Y}_{L M M}^{S H}$ shows the same phenomena.

In addition, since practically we have no true values of small areas, the comparison of each estimators can be conducted by using the comparison statistics such as regression methods, coverage and calibration. These practically used comparison statistics are studied by Brown et al. (2001). In this study, instead of using those comparison statistics, we simply calculate simple correlation coefficients between $\hat{Y}_{D E}$ and the other estimators in order to check closeness to $\hat{Y}_{D E}$. The results are shown in Table 3.2 and show that $\hat{Y}_{L M M}$ is better than $\hat{Y}_{L O G I T}$. The shrinkage estimators corresponding to the original estimators have almost the same correlation coefficients.

\subsection{Simulations}

In order to compare the efficiency of these estimators, we conduct a small simulation study. First we select samples without replacement from the whole samples with sample size 2,000, 3,000 and 4,000. We replicate 1,000 times to obtain the comparison statistics. For comparison, five comparison statistics are used. These are Mean Squared Error(MSE), Relative Mean Squared Error(RMSE), Mean Absolute Error(MAE), Absolute Relative Error(ARE), and Relative Bias(RB). Details about these comparison statistics are presented in Rao (2003). Here, $Y_{i}$ is the true value for small area $i$, and each estimate of $Y_{i}$ is $\hat{Y}_{i}$.

$$
\begin{array}{rlrl}
\mathrm{MSE} & =\frac{1}{n R} \sum_{r=1}^{R} \sum_{i=1}^{n}\left(\hat{Y}_{i, r}-Y_{i}\right)^{2}, & \mathrm{RMSE}=\frac{1}{n R} \sum_{r=1}^{R} \sum_{i=1}^{n}\left(\frac{\hat{Y}_{i, r}-Y_{i}}{Y_{i}}\right)^{2}, \\
\mathrm{MAE} & =\frac{1}{n R} \sum_{r=1}^{R} \sum_{i=1}^{n}\left|\hat{Y}_{i, r}-Y_{i}\right|, & \mathrm{ARE}=\frac{1}{n R} \sum_{r=1}^{R} \sum_{i=1}^{n}\left|\frac{\hat{Y}_{i, r}-Y_{i}}{Y_{i}}\right|, \\
\mathrm{RB} & =\frac{1}{n R} \sum_{r=1}^{R} \sum_{i=1}^{n} \frac{\left(\hat{Y}_{i, r}-Y_{i}\right)}{Y_{i}} . & &
\end{array}
$$

Here $n=34$ is the number of small areas and $R=1,000$ is the number of replications. To use the above statistics, we need to know the true values, $Y_{i}, i, \ldots, n$ in each small area. However practically we have no true values. So in this simulation, we just assume that the estimates of total in each small area obtained using $\hat{Y}_{D E}$ with the whole data as the true values shown in Table 3.1. This is the same way used in Hwang and Shin (2009). The comparison results of estimators are tabulated from Table 3.3 to Table 3.5 . Here we drop $\hat{Y}_{D E}$ in this comparison since only $\hat{Y}_{D E}$ is a design-based estimator. Notice that MSE and MAE are the statistics about the size of error with RMSE and ARE as the statistics about the size of relative error.

From Table 3.3 to Table 3.5, we have the following results. As expected, values of MSE and MAE of shrinkage estimators, $\hat{Y}_{L O G I T}^{S H}$ and $\hat{Y}_{L M M}^{S H}$ are larger than those of $\hat{Y}_{L O G I T}$ and $\hat{Y}_{L M M}$ respectively. However, for RMSE and ARE, the values of shrinkage estimators, $\hat{Y}_{L O G I T}^{S H}$ and $\hat{Y}_{L M M}^{S H}$ have reverse results. Specially, note that for MSE, $\hat{Y}_{L O G I T}$ and $\hat{Y}_{L O G I T}^{S H}$ have almost the same results whereas 
Table 3.3. Results on comparison statistics with sample size 2,000

\begin{tabular}{lccccc}
\hline \multicolumn{1}{c}{ MSE } & RMSE & MAE & ARE & RB $(\%)$ \\
\hline$\hat{Y}_{L O G I T}$ & 8961892 & 0.490 & 2216 & 0.499 & 0.232 \\
$\hat{Y}_{L M M}$ & 7681710 & 0.347 & 2004 & 0.428 & 0.161 \\
$\hat{Y}_{L O G I T}^{S H}$ & 8972775 & 0.432 & 2203 & 0.475 & 0.178 \\
$\hat{Y}_{L M M}^{S H}$ & 8317045 & 0.257 & 2055 & 0.39 & 0.012 \\
\hline \multicolumn{7}{r}{ Table 3.4. Results on comparison statistics with sample size 3,000 } & & & \\
\hline \multicolumn{7}{c}{ MSE } & RMSE & MAE & ARE & RB $(\%)$ \\
\hline$\hat{Y}_{L O G I T}$ & 8472503 & 0.468 & 2164 & 0.491 & 0.238 \\
$\hat{Y}_{L M M}$ & 6432409 & 0.273 & 1814 & 0.383 & 0.134 \\
$\hat{Y}_{L O G I T}^{S H}$ & 8554284 & 0.393 & 2153 & 0.459 & 0.167 \\
$\hat{Y}_{L M M}^{S H}$ & 7418413 & 0.207 & 1917 & 0.356 & -0.020 \\
\hline
\end{tabular}

Table 3.5. Results on comparison statistics with sample size 4,000

\begin{tabular}{lcccrr}
\hline & MSE & RMSE & MAE & ARE & RB(\%) \\
\hline$\hat{Y}_{\text {LOGIT }}$ & 8149336 & 0.448 & 2126 & 0.483 & 0.236 \\
$\hat{Y}_{\text {LMM }}$ & 5566040 & 0.222 & 1678 & 0.348 & 0.106 \\
$\hat{Y}_{L H}^{S H I T}$ & 8210022 & 0.393 & 2119 & 0.459 & 0.182 \\
$\hat{Y}_{\text {LM }}^{S H}$ & 6403534 & 0.171 & 1772 & 0.326 & -0.038 \\
\hline
\end{tabular}

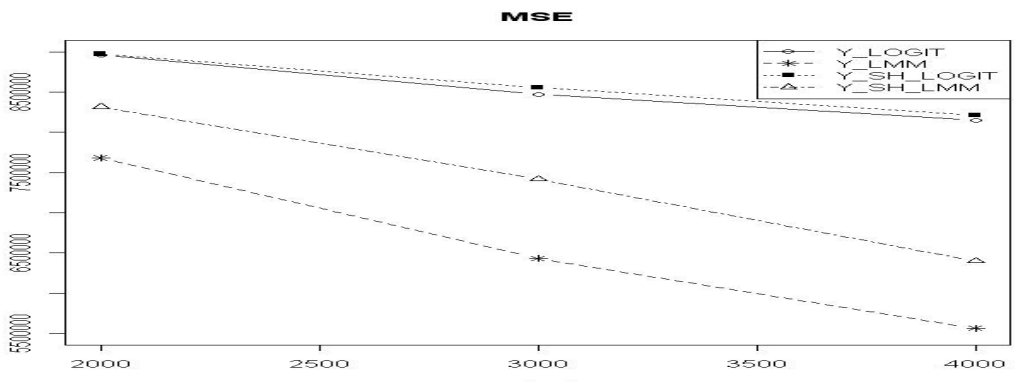

Figure 3.1. MSE results

for RMSE, the difference is large. In addition, $\hat{Y}_{L M M}$ and $\hat{Y}_{L M M}^{S H}$ provide the best results in all comparison criteria. For checking unbiasedness, RB is considered and $\hat{Y}_{L M M}^{S H}$ shows the best results. The results show that shrinkage estimators have some bias; however, the values are not large. The shrinkage estimators are shown to produce better results in RB than the corresponding original estimators.

To investigate the trend of magnitude of errors as sample sizes increase, we draw figures of MSE, RMSE, and RB.

From Figure 3.1 and Figure 3.2, the results on MSE and RMSE, we find that as the sample size increases, $\hat{Y}_{L M M}$ and $\hat{Y}_{L M M}^{S H}$ quickly reduce the magnitude of errors and magnitude of relative errors relatively to $\hat{Y}_{L O G I T}$ and $\hat{Y}_{L O G I T}^{S H}$. Figure 3.3 , the results on RB, shows that even though $\hat{Y}_{L M M}^{S H}$ provide good results, four estimators do not reduce the relative bias as sample size increases.

\section{Conclusion}

For the small area estimation of binary response data such as un-employment, logistic regression estimator and logistic mixed estimator are widely used as they have some good statistical proper- 


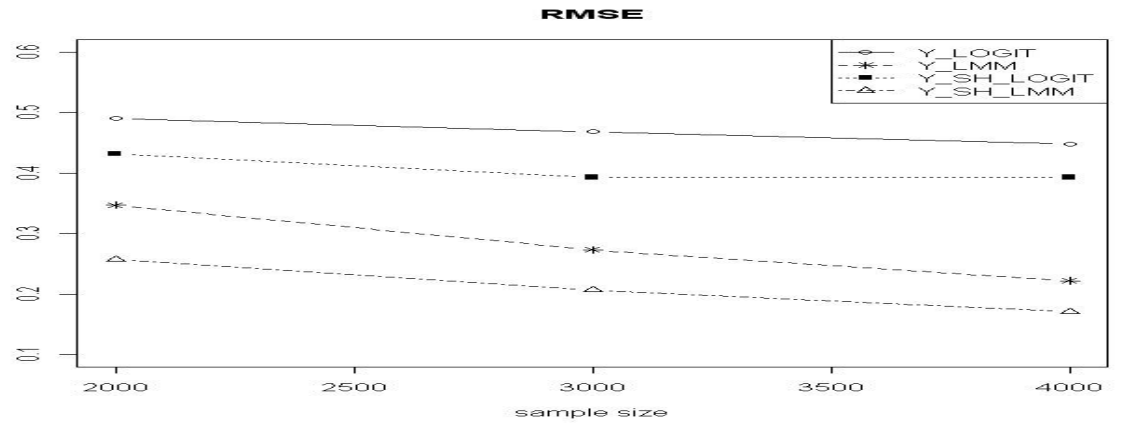

Figure 3.2. RMSE results

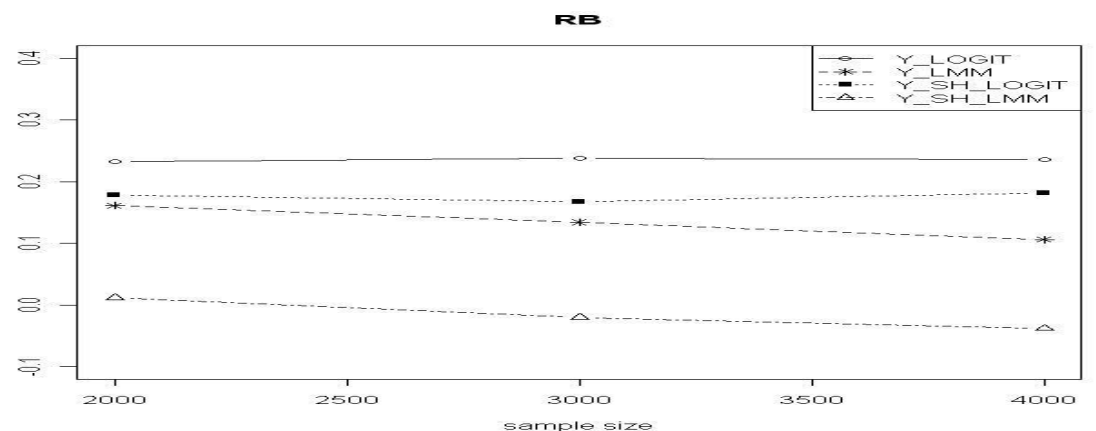

Figure 3.3. RB results

ties. These estimators are obtained by minimizing MSE. However, relative error criterion should be considered and applied to obtain the better small area estimation in some cases where the interpretation of analysis results is considered as a primary interest rather than the precision of the estimator.

In this paper we study logistic regression type shrinkage estimators obtained by minimizing $\mathrm{RE}$ and compare them with logistic regression and logistic mixed estimators. Comparison results based on MSE show that $\hat{Y}_{L M M}$ is superior to any other estimators including $\hat{Y}_{L O G I T}$. However $\hat{Y}_{L M M}^{S H}$ shows the best results in RMSE criterion. Therefore we conclude that two small area estimators, $\hat{Y}_{L M M}$ and $\hat{Y}_{L M M}^{S H}$ provide the best results according to proper situations. If an analyst concludes that MSE is a more important criterion than RE, $\hat{Y}_{L M M}$ should be used; however, in the opposite case, $\hat{Y}_{L M M}^{S H}$ should be used.

\section{References}

Agresti, A. (2002). Categorical Data Analysis, John Wiley and Son, New York.

Brown, G., Chambers, R., Heady, P. and Heasman, D. (2001). Evaluation of small area estimation methods - Application to unemployment estimations for U.K. L.F.S., Proceedings of Statistics Canada Symposium 2001.

Hwang, H.-J. and Shin, K.-I. (2008). Shrinkage prediction for small area estimations, The Korean Journal of Applied Statistics, 21, 109-123.

Hwang, H.-J. and Shin, K.-I. (2009). A small area estimation for monthly wage using mean squared percentage error, The Korean Journal of Applied Statistics, 22, 403-414. 
Jeong, S. O. and Shin, K.-I. (2008). A new nonparametric method for prediction based on mean squared relative error, The Korean Communications in Statistics, 15, 255-264.

Kim, Y.-W. and Choi, H.-A. (2004). Small area estimation technique based on logistic model to estimate unemployment rate, The Korean Communications in Statistics, 11, 583-595.

Park, H. and Stefanski, L. A. (1997). Relative error prediction, Statistics and Probability Letters, 40, 227236.

Rao, J. N. K. (2003). Small Area Estimation, John Wiley and Son, New York.

Yeo, I.-K., Son, K. and Kim, Y.-W. (2008). Small area estimation via generalized estimating equations and the panel analysis of unemployment rate, The Korean Journal of Applied Statistics, 21, 665-674. 